\title{
What Role Does the Authoritarian Leadership and Benevolent Leadership Play in the Relationship Between Voice Behavior and Innovative Behavior?
}

\author{
Ping Wang ${ }^{\mathrm{a}, *}$ and Shaoqi Wang ${ }^{\mathrm{b}}$ \\ School of Economics and Management, Zhejiang Sci-Tech University, Hang Zhou, China, 310018 \\ àw190923@163.com, ${ }^{\text {b } 15858133483 @ 163 . c o m ~}$ \\ *corresponding author
}

Keywords: Voice Behavior, Innovative Behavior, Authoritarian Leadership, Benevolent Leadership

\begin{abstract}
When individuals can actively and proposing suggestions, they will also show a stronger desire for innovation. The generation of voice behavior can promote the creation of individual innovative behavior. In the background of Chinese culture, this process will be influenced by authoritarian leadership and benevolent leadership. Authoritarian leaders play a negative regulatory role in the process of voice behavior influencing innovative behavior, while benevolent leadership plays a positive regulatory role.
\end{abstract}

\section{Introduction}

With the transformation of made in China into created in China, innovation has been repeatedly mentioned. Chinese companies need to have enough energy and innovation to stay competitive in a rapidly changing market, and enterprise innovation is based on individual employee's innovation. Innovative behavior can be seen three stages of view which as the process of producing, implementing and completing(Scott\&Bruce,1994).The factors influencing the employee's innovative behavior include the self and external factors, and the self-influencing factors such as the voice behavior promote the creation of individual innovative behavior(Dyne, Ang, Botero, 2003).Voice behavior is an act of expressing own innovative proposals and suggestions for improvement (LePine, Van Dyne,1998), which itself is an individual innovative behavior.

In addition, individual behavior is largely influenced by the leadership style. Being a good leader, encouraging employees to innovate is one of their necessary capabilities ( $\mathrm{X}$ Zhang, KM Bartol,2010).There are also significant differences in the leadership styles formed under different cultural backgrounds. In the cultural background of China, paternalistic leadership can better explain the organizational behavior and effectiveness of Chinese companies than Western leadership theories (Zong-You Wu,Wei Xu,Bo-Ying Zheng, 2002).There is a sharp contrast between the style of authoritarian leadership and benevolent leadership. authoritarian leadership emphasizes control, and benevolent leadership emphasizes concern, the effects of the two are diametrically opposed (Wu Min, Huang Xu et al., 2007;Duan Jinyun, 2012; Huang Xu, 2012 ).Therefore, both of them have different roles in the process of voice behaviors that affect innovative behavior.

\section{Theoretical Basis}

\subsection{What is Voice Behavior?}

Voice behavior is a change-oriented approach to improving the environment and is a constructive interpersonal behaviour (Borman,Motowido,1997;Jin-Yun DUAN, Jia-Nan ZHONG, 2005).Its purpose is to provide ideas or perspectives and to generate positive daring challenges and constructive improvements in order to accomplish the task of a team or organization and to improve organizational performance(De Dreu et al.,2001;Detert ,Burris,2007;Liu, Zhu, Yang,2010).

Many studies show that the act of voice behavior is not a single dimension but a 
multidimensional and complex concept (Withey,Cooper,1989).Divided by motivation as Conscious voice behavior, unconscious voice behavior, and conscious silent behavior(Hischman,1970); or divided into pro social voice behavior, defensive voice behavior, and acquiescence of behavior(Van Dyne, Ang, Botero,2003).There are also some scholars divide the object of voice into two groups: executive director and direct chief executive (Ioannis etal.,2008);or voice to colleagues and voice to superiors (Liu etal.,2010).Liang\&Farh(2008)rely on the previous research and Chinese background, the behavior is divided into promotive voice and prohibitive voice. According to the doctrine of the mean of Chinese traditional culture, another scholar divided the voice behavior into self-aggressive voice and considerate the overall situation of voice(Jin-Yun DUAN, Bin LING,2011).

Due to the diversity of the dimensions of voice behavior, the measurement methods are also different. Such as the Hagerdoorn et al. (1999) development of the caring voice and aggressive voice two-factor scale. On the basis of foreign studies and combined with the background environmental factors in China,Liang\&Farh(2012) developed a two-dimensional voice behavioral questionnaire for both promotive and prohibitive. At present, this scale is the most widely used measurement scale by domestic scholars(Rui LI, Wen-Quan LING, Shi-Shun LIU,2009;Rui LI, Wen-Quan LING, Li-Luo FANG,2010;Chang-Chun XIANG, Li-Rong LONG,2013; Jian LIANG,2014).

\subsection{What is Innovative Behavior?}

From the individualistic perspective, innovation is a capability and willingness. For example, Kirton (1976) argued that innovative behavior is a way to break the routine and take a fresh approach to solve problem .Hurt etal.(1977)argued that personal innovation should be defined as the willingness to change. However, most scholars believe that innovation is a process. Vandeven(1986) argued that innovation was based on the practice of developing, implementing, reacting and amending the realization of innovative ideas. The essence of innovation lies in the innovation of ideas.Personal innovation is a complex process, which can be divided into three stages: the first stage is the individual contact problem and have a cognition, the second stage is the innovation individual seeks supporters, the third stage is the individual visualizes their creativity to get the prototype of innovation (Kanter, 1988).In addition, innovative behavior can be regarded as the process of generating, implementing and completing the three phases(Scott\&Bruce,1994).Many Chinese scholars also agree with this theory (Xiao-Jun LU,Guo-Liang ZHANG,2007;Yuan-Dong GU,Ji-Sheng PENG,2010).

At present, the most commonly used dimension of innovative behavior and measurement scale is the single-dimension scale which is developed by Scott \& Bruce (1994). The scale also has a high reliability and validity in the study (Jing-Ji WU,He-Mao LIN,1998;Ting-Ting ZHANG,2009;Yun LIU, Jin-Tao SHI,2009).Based on this scale, Zhou\&George(2000)later adapted a 13-item singledimension scale, which was mainly measured by managers' evaluation of employee's individual innovative behavior.

\subsection{What are Authoritarian Leadership and Benevolent Leadership?}

Authoritarian leadership and benevolent leadership are derived from paternalistic leadership. BoXun Zheng(1995) proposes a dualistic model of paternalistic leadership. Paternalistic leadership is divided into mercy and authority. In the subsequent study, the model was modified again, adding the dimension of morality, and finally obtaining a tri-modal model that included authoritarian leadership, benevolent leadership, and moral leadership(Bo-Xu Zheng,Jing-Li Fan,2000).

Authoritarian leadership is to control the subordinates and maintain their own authority. The specific performance of the behavior has the right to work style, derogatory subordinates, image decoration and teaching behavior. Subordinates will show respect and obedience to leaders. Benevolent leadership refers to the ability of leaders to take care of employees, show concern for them, and maintain the Mianzi of employees, in order to promote subordinates' gratitude and improve their own work (Bo-Xu Zheng, Jing-Li Fan,2000).

With the introduction of the paternalistic leadership ternary model, combined with the CPM scale (Wen-Quan Ling,1987),Bo-Xun Zheng etal(2000)added the C scale which as the moral 
leaderships scale to the binary scale. Finally, a three-dimensional measure scale PLS scale was formed. After empirical research, it was further optimized into a 28-item scale that included 11 benevolent leadership items, 9 moral leadership items, and 8 authoritarian leadership items (Bo-Xun Zheng,2004).

\subsection{The Relationship between Voice Behavior and Innovative Behavior}

Innovative behavior itself is a challenge to the existing work processes and presents new and valid ideas when problems are discovered (LePine, Van Dyne, 1998).Voice behavior can be divided into promotive voice behavior and repressive voice behavior. Promotive voice behaviour is that employees express their views and new ideas in order to improve the performance of organizations or teams (Liang, Farch, 2012).In the course of innovation behavior, employees will firstly put forward their ideas and then exchange ideas with colleagues or leaders. Combined with promotive voice behaviour, it is not difficult to find that promotive voice behaviour itself is a individual behaviour which has innovative tendency(Li-Li LIU,2017).

Many studies show that voice behavior can enhance employees' innovative awareness and ability, thus promoting their innovative behavior. De Dreu \& Van Vianen(2001)argued that the individual voice behavior of employees can stimulate innovative behavior. Employees can introduce their ideas to organizational members or seek new ways to solve problems in order to achieve better performance. Voice behavior is an organizational behavior that activates innovative ability, and has a direct relationship with the emergence of innovative behaviour (Dyne,Ang,Botero,2003). Employees' knowledge is important for innovation (Amabile, 1994),Lynch (2007) emphasizes the importance of employees' knowledge to organizational innovation. He believes that organizations need to have the ability to utilize employees' knowledge, which is closely linked with voice behavior. Employees' voice behavior level will have a significant impact on team's innovation performance, which will affect employee's individual innovation behavior(Gong-Min BAO, YuanYuan QIAN, 2009).Ng\&Feldman (2012) found that the voice behavior not only has a positive effect on the performance of employees, but also has a strong predictive effect on employee's individual innovative ability and implementation of innovative ideas.

As a result, it can be speculated that the voice behavior has a positive predictive effect on innovative behavior.

\section{Theoretical Model}

Individual behaviors are basically affected by the influence of individuals and the environment. Therefore, innovative behavior can also be studied from two factors, the individual and the environment (Pan-Pan Wang,2016).Situational factors play an important role in the impact of personality on performance(Tett\&Bumet,2003).From the perspective of the environment, leadership styles of leaders(Walumbwa FO,2008;Ru-Jie Qu,2014), organizational culture, the organizational climate and so on will have an impact on individual behavior.

The authoritarian leaders will undermine exclusive rights and strict control, which will undermine the internal interactions within the organization (Bo-Xun Zheng etal.,2002;Ang Gao etal.,2014).This will lead to employees not learning from each other and making suggestions, so that the knowledge and skills will not be improved. This has hindered the creation of innovative behavior; in addition, authoritarian leaders require subordinates to obey absolutely, so that subordinates will not tend to voice, and fear that words and sentences will cause adverse effects, and therefore will lose many opportunities to present innovative ideas. Therefore, it can be speculated that authoritarian leaders have a negative regulatory effect on the relationship between voice behavior and innovative behavior.

Benevolent leadership through meticulous care for subordinates, so that subordinates will generate gratitude and return (Cheng,2004,2006).Therefore, when the benevolent leader expresses concern for the subordinate's work and life, the employee will devote more energy to work and will also make the employee produce more innovative thinking(Ang Gao etal.,2014).And benevolent leadership can tolerate team differences and conflicts, greatly promote the exchange and sharing 
among the members of the organization; In addition, in the style of benevolent leadership, the subordinates dare to express their opinions to voice and play a innovative mind( $\mathrm{Li}-\mathrm{Li}$ Ma,2014).Therefore, it can be speculated that benevolent leadership has a positive regulatory effect on the relationship between voice behavior and innovative behavior.

According to the above analysis, we can get the theoretical model, shown in Figure 1:

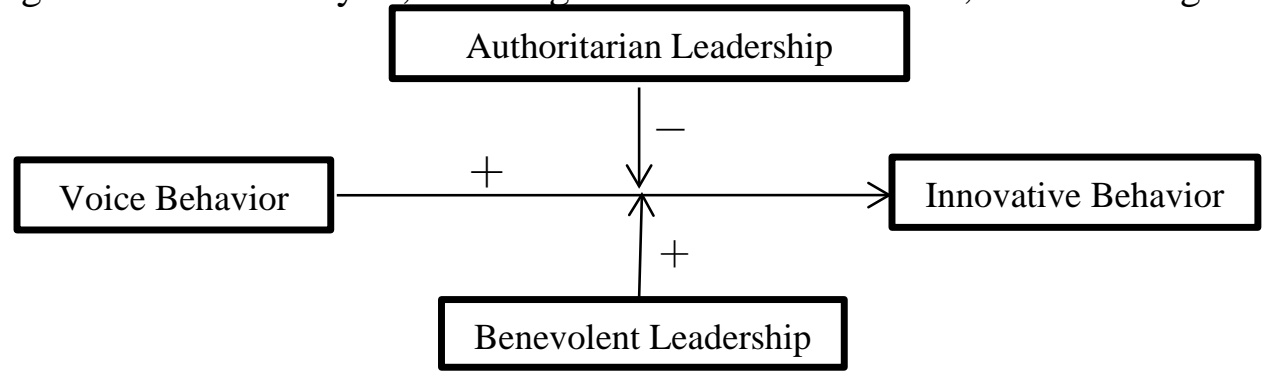

Figure 1 Theoretical Model

\section{Summary}

Employees are also motivated by their innovative behaviors in the process of voice behaviors. In addition, as the authoritarian leaders and benevolent leaders under the background of traditional Chinese culture, the roles played by the leaders in the process of voice behavior influence innovative behavior are not the same. The authoritarian leader play a negative adjustment role, and the benevolent leadership plays a positive role.

Therefore, enterprises should attach great importance to incentives towards voice behaviour and innovative behaviour in the process of management. In the cultural background of China, Chinese cares a lot about the opinions of others and social evaluations .Chinese employees also think highly of leaders' ideas and colleagues' evaluation. In order to the appreciation of the leaders, employees will have more voice behaviors and innovative behaviors. On the contrary, employees who are afraid of leadership and punishment will make employees not to make suggestions and innovations, as suggestions and innovations may change the status quo, or harm the interests of others. In order not to conflict with others, they will choose to maintain the status quo instead of making voice behaviour and innovative behaviour. Under the leadership of benevolent, the employees will have a free environment to work, and they will have a mentality of knowing and reporting for the leaders, encourage employees to exert their personal initiative, provide advice for the development of departments and enterprises, and actively generate innovative behaviors. Therefore, leaders need to exhibit more benevolent leadership styles in order to promote the production of voice behaviors and innovative behaviors. Under the authoritarian leadership, employees will develop a kind of obedient work attitude that is not conducive to their own development and innovation.

In addition to the birth of innovative ideas, the key to innovative behavior of employees lies in the realization of innovative ideas. Therefore, creating an environment conducive to employees' innovative ideas, companies need to provide favorable conditions to help employees achieve their own innovative ideas. Leaders should take meticulous care of their employees and solve their difficulties in their work and life so that their subordinates have no worries. Under such conditions, employees can more easily obtain resources for realizing innovative ideas and have a positive effect on the creation of individual innovation behaviors.

\section{References}

[1] Scott,S.G., Bruce, R.A..(1994).Determinants of innovative behavior: A path model of individual innovation in the workplace,[J].Journal of Academic management,,37 (3) :580-607.

[2] Van Dyne,L.,Ang,S.,Botero,I.C.(2003).Conceptualizing employee silence and employee voice as multidimensional constructs[J].Journal of Management Studies,40,1359-1392.

[3] LePine J A,Van Dyne L.(1998).Predicting voice behavior in work groups [J].Journal of Applied 
Psychology,83(6):853-868.

[4] Zhang X,Bartol K M.(2010).The influence of creative process engagement on employee creative performance and overall job performance: A curvilinear assessment[J].Journal of Applied Psychology,95(5):862-873.

[5] Zong-You Wu,Wei Xu,Bo-Xun Zheng.(2002).Relationship between authoritarian leadership behavior and subordinates' anger emotional response in an uncontrollable or unbearable Chinese enterprise[J]. Native Psychology Research,(18):13-50.

[6] Jin-Yun Duan.(2012).The influence of paternalistic leadership on the behavior of employee's speech and psychological security. [J]. Management Review,24(10), 57-60.

[7] Xu Huang, Kan Shi, Li-Ping Gao,Yue-Jia Sha.(2012).The influence of leadership authorization behavior on employee's silence: analysis of the regulatory role of trust [J].Management Review,24(10):94-101.

[8] Borman,W.C.,Motowidlo,S.J.(1997).Task performance and contextual performance: The meaning for personnel selection research[J].Human performance,10(2),99-109.

[9] Detert,J.R.,Burris E.R.(2007).Leadership behavior and employee voice: Is the door really open?[J]. Academy of Management Journal,50(4), 869-884.

[10] Liu W,Zhu R,Yang Y.(2010).I warn you because I like you: Voice behavior, employee identifications, and transformational leadership[J]. Leadership Quarterly,21(1), 189-202.

[11] Kirton,M.J.(1976).Adaptor and innovators:A description and measure [J].Journal of Applied Psychology,,61:622-629.

[12] Hurt,H.T.,Joseph K\&Cook C.D..(1977)Scales for the measurement of innovativeness [J]. Human Communication Research,4(1):58-65.

[13] Vandeven,A.H.(1986).Central problems in the management of innovation [J].Management Science,32:590-607.

[14] Kanter,R.M..(1988).Structural collective and social conditions for innovation in organization [J].10:169-211.

[15] Bo-Xun Zheng, Li-Fang Zhou,Jing-Li Fan.(2000).Paternal Leadership Scale: Construction and Measurement of the Three Elements Model[J]. Native Psychology Research, (14),3-64.

[16] De Dreu C K W,Van Vianen A.E.M.(2001).Managing relationship conflict and the effectiveness of organizational teams [J].Journal of Organizational Behavior,22(3):309-328.

[17] NgT WH, Feldman D C.(2012).Employee voice behavior: A meta-analytic test of the conservation of resources framework[J].Journal of Organizational Behavior,33(2): 216-234.

[18] Tett R P, BumetD D.(2003).A Personality trait-based interaetionist model of job Perofmrnaee. Jounral of Applied Psyeholog, 88: 500-517.

[19] Ru-Jie Qu, Hai-Qin Kang.(2014).Research on the Impact of Leadership Behavior on Employees' Innovation[J]. Management Review,26(1): 88-98.

[20] Bo-Xun Zheng, Pei-Jun Xie,Li-Fang Zhou.(2002).Headmaster's style of leadership, quality of relationship, and teacher's role outside behavior: effects of transitional and paternalistic leadership [J]. Native Psychology Research.

[21] Ang Gao, Qing Qu, Bai-Biao Yang, Xiao-Shen Zhao.(2014).Research on the influence of paternalistic leadership on team work performance - Potential role of leadership ability[J].Science of Science and Management of S.\&T.,(1)100-106. 